\title{
The Next Generation Internet of Things Architecture Towards Distributed Intelligence: Reviews, Applications, and Research Challenges
}

\author{
Baha Rababah ${ }^{1}$, Tanweer Alam ${ }^{2}$ and Rasit Eskicioglu ${ }^{1}$ \\ ${ }^{l}$ Department of Computer Science, University of Manitoba, Canada. \\ ${ }^{2}$ Faculty of Computer and Information Systems. Islamic University of Madinah, Saudi Arabia. \\ baha@cs.umanitoba.ca
}

\begin{abstract}
Increasing the implication of growing data generated by the Internet of Things (IoT) brings the focus toward extracting knowledge from the raw data derived from sensors. In the current cloud computing architecture, all the IoT raw data are transmitted to the cloud for processing, storage, and controlling things. Nevertheless, the scenario of sending all raw data to the cloud is inefficient as it wastes the bandwidth and increases the network load. This problem can be solved by providing IoT Gateway at the edge layer with the required intelligence to gain the knowledge from raw data to decide whether to actuate or offload complicated tasks to the cloud. This collaboration between the cloud and the edge is called distributed intelligence. This work highlights the distributed intelligence concept in IoT. It presents a deep investigation of distributed intelligence between the cloud and the edge layers under IoT architecture, with an emphasis on its vision, applications, and research challenges. This work aims to bring the attention of IoT specialists to distributed intelligence and its role to deduce current IoT challenges such as availability, mobility, energy efficiency, security, scalability, interoperability, and reliability.
\end{abstract}

Index Terms - Cloud Computing; Distributed Intelligence; Edge Computing; Internet of Things; Machine Learning; Smart Gateway.

\section{INTRODUCTION}

It is expected that 50 billion smart devices will be associated with the Internet [1] and the generated data will be around 500 zettabytes by 2020 [2]. Recently, IoT becomes popular due to the development of Radio Frequency Identification (RFID), Wireless Networks, Bluetooth Low Energy (BLE), and sensing and actuating technologies. However, since most of the IoT applications are cloud-based, they have many challenges that cannot be ignored such as availability, energy efficiency, security and privacy, scalability, interoperability, and reliability. As the cloud-based applications, the sensor data of the IoT are processed and stored on distance servers hosted on the Internet, rather than a local server. Recently, scholars are trying to solve these challenged by looking at edge computing as it is closer to end devices (e.g., smart devices and sensors) than the cloud [3]. Edge computing means to enable the technologies that allow computing to be done at the edge of the network, close to the end devices. Processing data close to the end devices will help to solve the problems of latency, security, and privacy, availability and power consumption. Edge computing has the advantages of fast processing, energy efficiency, security, mobility, and heterogeneity. On the other hand, cloud computing cannot be omitted, as edge computing has limited process and storage capability.

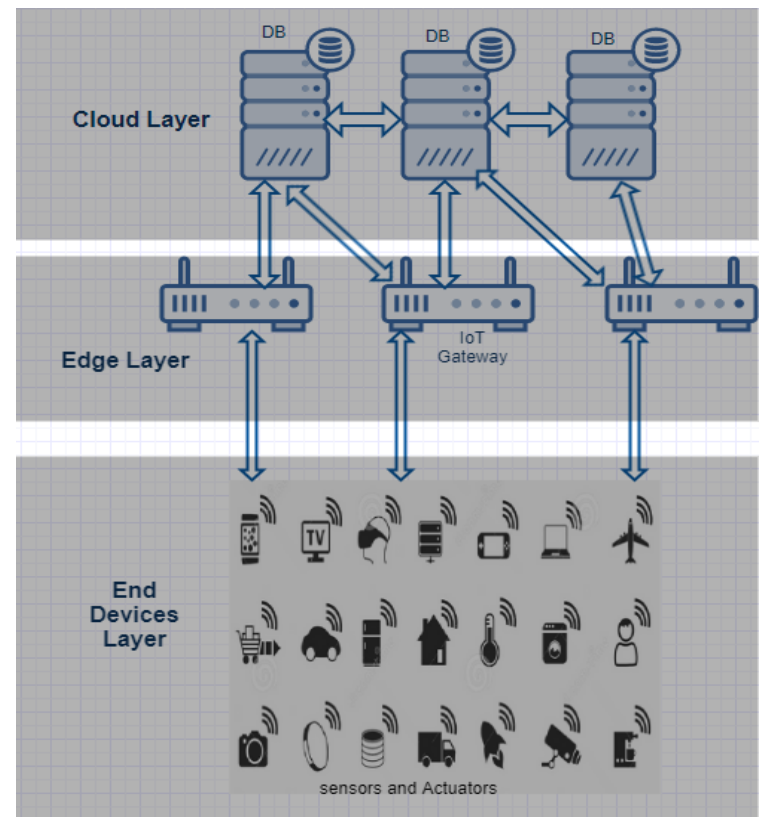

Figure 1: IoT and cloud structure

Cloud computing has the advantages of large storage space, processing capabilities, data analysis, and easy access [4]. Therefore, integrating edge and cloud in one model, that is combining their features in one model for supporting IoT applications is essential.

The current structure that combines IoT and cloud consists of three layers: cloud layer, edge layer, and end devices layer, as shown in Figure 1. The cloud layer consists of a group of connected powerful computers that can process huge and complex data. The edge layer is the middle layer between the cloud and end devices that sequel the advantages of cloud computing by offering extra services for the requirements of IoT. The last layer is the end devices layer that consists of sensors and actuators (things). In general, sensors collect the raw data from the environment and send it to the edge layer. The Gateway at the edge layer pre-processes that data by connecting cloud and end devices by different transmission protocols and forwards the data to the cloud. The cloud processes the data to obtain the Knowledge and makes the decision. Thereafter, it sends the decision to the actuators at the end devices layer through the edge layer. Sending a huge amount of data to the cloud and return with the decision is an 
inefficient way that causes latency, availability, security, and privacy, and power consumption problems. The current network architectures and technologies are not sufficient to send a huge amount of data to the cloud and return with the decision.

Nowadays, scholars are investigating processing IoT data close to the end devices layer to avoid the challenges. Many papers suggested building IoT gateway at the edge layer that can process the raw data and make the decision without sending the data to the cloud. Single-board computers, such as Arduino and Raspberry Pi have acceptable computational capabilities that make them able to act as IoT gateway at the edge layer to process the raw data to make the decision [5]. However, those constrained devices do not have the same computational power of the cloud to deal with the huge amount of IoT data. In this case, distributing the intelligence between edge and cloud becomes necessary. We can define Distributed Intelligence in IoT as a paradigm that uses models, techniques, and algorithms for deciding whether to process IoT data in the edge layer, cloud layer, or both together [6-8].

Accordingly, this work highlights the role of Distributed Intelligence models that combines the advantage of both edge and cloud layers. Distributed Intelligence distributes the intelligence over the edge and cloud layers to provide the required functionality and performance for IoT applications [9]. It will enable the IoT gateway to extract most of the knowledge from the sensors' data to decide without sending unnecessary data to the cloud. Processing and deciding by IoT gateway instead of cloud will solve the problems of latency for real-time applications. In some cases, when the gateway is unable to process the data and make the decision, the cloud will make the decision. For example, when turning on the heating system at a smart home, the sensors will collect the data of temperature, time and the motion at home, and then send it to the IoT gateway at the edge layer. The gateway will process the data, decide to turn on/off the heating system and send the decision back to the heating system at the home. However, if the gateway is unable to make the decision (e.g the task is too complex, or the gateway is unable to process the task), the gateway will forward the data to the cloud layer. For example, if a video processing application consumes the CPU of the gateway, the task might be offloaded to the cloud layer to be processed. This paradigm helps the gateway to still under load.

This paper is organized as follows. In the second section, we introduce the related work related to Distributed Intelligence in IoT. The following section takes a close look at the Internet of Things, cloud computing, and edge computing respectively. The fourth section represents the Distributed Intelligence in IoT. In the fifth section, we introduce some of IoT applications that highlight the need for Distributed Intelligence. In the last section, we represent the main current challenges of enabling Distributed Intelligence in IoT. To the best of our knowledge, this paper presents the first comprehensive review of Distributed Intelligence in IoT to date.

\section{RELATED WORKS}

In 1990, the concept "Internet of Things" was firstly introduced by Kevin Ashton, the Executive Director of AutoID Labs at MIT, while giving a talk for Procter \& Gamble [10]. During his talk, he stated that all the data available on the web were first produced by humans who have limited attention, time and accuracy. He added that we need computers that know everything about things using gathered data without any help from humans. Later on, IoT has been evolved into a system that uses a variety of technologies, such as the Internet, wireless communication, microelectromechanical systems, and embedded systems.

Nowadays, extracting knowledge from the collected raw data is one of the IoT challenges. IoT collects raw data from the sensors, sends them to the cloud for further processing. The cloud processes the data for decision making and sends it to the actuators. Many proposals applied the scenario of sending collected data from the environment to the cloud to store, manage, and make decisions [11-15], as it provides large storage, complex processing, and access anywhere/ anytime [16]. Unfortunately, cloud-based systems cause a high level of latency for IoT applications [17-19]. Therefore, researches started to look at the edge computing to process the data close to the things as it provides fast processing, energy efficiency, reliability, and security and privacy [20]. However, it is not able to offer large storage, complex processing, and access anywhere/anytime that the cloud offers. Therefore, the solution is to integrate the edge and cloud computing to realize both advantages for IoT applications.

In the last decade, many kinds of research discussed and proposed models of IoT and cloud integration [21-24]. Researches in $[21,22]$ demonstrated the need to integrate IoT with the cloud by presenting a deep understanding of the integration between cloud and IoT [25]. They also provided an overview of the current researches related to this topic. Additionally, they mentioned the challenges and solutions points of current approaches. Babu et al. [23] presented an architectural design for integration of the cloud and IoT. Li et al. [24] employed the TOSCA standard for cloud service management to systematically specify the components and configurations of IoT applications. TOSCA describes the internal topology of application components and the deployment process of IoT applications. All the previously mentioned models are cloud-based, which need to transfer all raw data to the cloud for processing and making decisions. None of the previously mentioned papers discussed processing raw data collected from end devices at the edge layer for the purpose of learning, making decision, and taking actions, without sending the data to the cloud.

A large amount of work has been performed in the field of enabling edge computing to process the generated raw data and to make decisions. Some of the works focus on enabling the IoT gateway at the edge of the network to process and manage the raw data. Mueller et al. [26] introduced a SwissQM/SwissGate system to program, deploy, and operate wireless sensor networks. They proposed a gateway called SwissGate and applied it to smart home applications. JongWang et al. [27] came up with a sensor network system that consists of one main server and several servers that act as gateways, and are connected to different sensor networks. Designing such a system requires a lot of configurations and high hardware costs. In another work, Bimschas et al. [28] came up with a smart gateway approach that is able to execute application code. They proposed a middleware for the gateway to run different applications, such as protocol translation, request caching, and intelligent caching with sensor discovery. For general IoT applications, Guoqiang et al. [29] came up with a general-purpose smart IoT gateway 
that supports several communication protocols to translate different sensor data and unified external interfaces fitting for flexible software development. To use smartphones as a gateway, Bian et al. [30] utilized the android phone as a temporarily used smart home gateway that can predict user behavior to shut down unused devices. This works aimed to provide a dynamic home gateway that can reduce the wasted energy of the smart home.

In healthcare IoT application domain, Shen at al. [31] presented an intelligence 6LoWPAN border router that connects the health care sensors with IP network and uses a hidden Markov Model to make local decisions of health states. Stantchev et al. [32] introduced three-tier architecture (cloud, gateway, and smart items) to enable servitization for smart healthcare infrastructure. Rahmani et al. [33] also suggested an intelligence e-health IoT gateway for remote health monitors systems at the edge of the network in a threelevel architecture. The gateway can provide several services such as real-time data processing, local storage, and embedded data mining. Azimi et al. [34] presented a hierarchical computing model for IoT-based health monitoring systems based on the MAPE-K [35] computing model, introduced by IBM. The model benefits of fog and cloud computing to partition and execute machine learning data analytics.

To counter the intelligence challenges in IoT gateway, Wang et al. [36] proposed a framework of smart gateway for smart homes that consists of a smart home layer, smart gateway layer, and cloud layer. While the gateway performs data collection, awareness, and reporting, the cloud stores the reported data, and adjusts the data collection and awareness policy. Rahman et al. [37] proposed a Distributed Intelligence model for IoT gateway based on a belief network concept to learn, predict, and make decisions. This system starts from a small number of predefined rules, and then the system can change the rules based on past experiences.

In recent years, a large effort has been performed in the field of IoT gateway that is located between the cloud and the end devices. Although there has been a large amount of work being done to enable the gateway at the edge layer, there is only a small improvement towards providing intelligence to the gateway by extracting the knowledge from the raw data at IoT gateway to make the decision locally. Furthermore, there have been a few types of research performed in collaborating between the IoT gateway and the cloud, when the gateway is unable to analyze data, make decision and take action.

\section{RELATED ASPECTS}

\section{A. Internet of Things}

The IoT is a paradigm to connect an unlimited number of objects with their unique identifications anytime and anyplace using the IoT communications protocols [38]. IoT objects might be mobile phones, clothing, machines, and doors. It has been involved in many fields such as smart home, intelligence health care [39], and intelligent transportation. The recent innovative developments of information and communication technologies related to ubiquitous communication [40], pervasive computing, and ambient [41] intelligence play an essential role in the emergence of IoT [42]. Ubiquitous Communication is a concept in communication and computer networks [43, 44], where interconnection can be made anywhere and anytime. Pervasive computing is the improvement in smart devices, including the computational feature process [45]. Ambient Intelligence [46] is the ability of objects to sense and respond to the physical environment [47]. The key technologies of IoT are discussed below.

\section{a. Radio-Frequency Identification (RFID)}

The RFID is the technology that uses radio waves to detect, identify and track an object electronically through a tag attached to the object. It can store and/or retrieve information using electromagnetic transmission and a radio frequency (RF) compatible integrated circuit. A simple RFID system consists of RFID readers and RFID tags [48]. The RFID tag can be read by the RFID reader [49] from up to several feet away and does not require to be tracked within a direct line with the reader. A reader can scan several tags at the same time and transfer it to the server [50, 51]. RFID technology is widely used with different IoT applications, such as smart home, public transportations, health services, and Smart Agriculture, to identify things electronically $[52,53]$.

\section{b. Wireless Sensor Network (WSN)}

A sensor is a device that is able to detect a certain type of input from the physical object or the environment such as heat, smoke, light, motion, and pressure. In general, the output of a sensor is an electrical signal that is transmitted to the data center for processing and extracting knowledge. WSN is a group of sensors that communicates wirelessly and hop-by-hop to gather information and to detect a certain event in a physical environment [54]. WSN provides different beneficial data that might be used in certain applications and services. However, it faces many challenges in terms of communication and resource constraints such as short communication range, mobility, reliability, and security.

\section{c. Addressing}

The development of the Internet has led to interconnect objects and intelligent systems. In this case, identifying and managing objects are very essential for IoT, as it consists of a large number of objects. Addressing adds more features to IoT, which are uniqueness, reliability, persistence, and scalability [33]. The current IPv4 is only able to identify a group of sensors based on their geographical location [55]. However, the current requirements for IoT are to identify every sensor individually based on its identification, location, or functions. IPv6 is able to achieve those requirements, as it has a large address size to address and manage IoT objects efficiently [56]. The IETF standardized adaption protocol for IPv6 is called 6LoWPAN [57]. It is represented by both RFCs 4944 and 6282.

6LoWPAN is a short form that combines Internet Protocol (IPv6) and Low power Wireless Personal Area Networks (LoWPAN). 6LoWPAN, therefore, provides end devices with restricted processing ability to transmit information wirelessly using an Internet Protocol. It allows compression techniques to compress the IPv6 and UDP packets after deleting excessive data from the header part [58].

\section{d. Middleware}

The middleware and application layer, with the main objectives of functional abstraction and device communication [59] plays a key role due to the heterogeneous objects , limited storage, and processing capabilities [60]. The middleware is categorized in layers such as object abstraction, service management, service composition and 
application [61]. In a generic IoT system, end devices usually collect specific information such as temperature, light, and pressure. This information passes through limited processing locally. Then, it is sent to the external gadget for computations. After that, it can be transmitted to the local area network until it reaches the Internet, then the cloud-based services [62]. The cloud-based service will convert the received data into knowledge after some processing operations [63]. This knowledge might need to be stored or action needs to be performed. To support IoT, middleware [64] should handle the following challenges: interoperability between heterogeneous devices, context awareness [65], managing data, privacy and security, and device discovery [66].

\section{B. Cloud Computing}

The concept "Cloud Computing" was firstly defined as "a model for enabling ubiquitous, convenient, on-demand network access to a shared pool of configurable computing resources (e.g., networks, servers, storage, applications, and services) that can be rapidly provisioned and released with minimal management effort or service provider interaction", $[42,67,68]$. Over the last few years, Cloud computing started to be popular in the IT industry, as it has the advantages of unlimited storage, complex processing capabilities, and access anywhere/ anytime [69]. It also provides virtual resources on demand. Leading IT companies such as Amazon, Microsoft, and Google have adopted this model to provide services over the Internet [70].

Cloud services are divided into three layers, as shown in Figure 2: Software as a Service (SaaS), Platform as a Service (PaaS), and Infrastructure as a Service (IaaS). SaaS delivers applications running on Cloud environments through the browser to customers [71]. PaaS refers to the operating environment including the operating system and software development frameworks. IaaS refers to the provision of instant computing infrastructure, storage, and network resources to manage the operating system, cloud applications, and cloud storage [72].

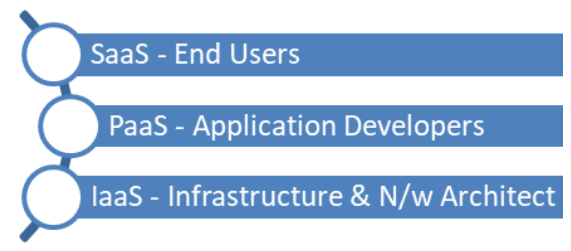

Figure 2: Cloud service models

\section{Edge Computing}

Edge computing is a paradigm of processing data at the edge of the network close to the end devices, where the data is generated, instead of sending the data to the cloud for processing [73]. The current Cloud architecture fails to handle the huge data generated by IoT devices, as it causes high latency and bandwidth utilization [74]. Thus, there is a need for edge computing to optimize computing in real-time IoT applications. The edge is a layer that is located between the cloud and the data source [75]. For example, a gateway in health care is the edge between health care things and the cloud. As shown in Figure 1, the things at the end devices layer can be data generated or consumers. Edge can perform several tasks such as data pre-processing, local data storage, data processing and caching, and request and deliver services from the cloud [76]. Edge computing has been found to improve the reliability, availability, and security and privacy of IoT applications, as the data is processed locally [77, 78].

\section{DiSTRIBUTED INTELLIGENCE IN IOT}

Distributed Intelligence in IoT is a paradigm that uses models, techniques, and algorithms for deciding whether to process IoT data in the edge layer, cloud layer, or both together. The development of IoT applications has recently become much easier with the availability of development kits, open hardware, and software. IoT Applications also become easy to be deployed on the cloud with the availability of Platform as a service (PaaS) resources and IoT cloud services. Between the cloud and the end devices (sensors), at the edge layer, gateway (computer board such as Arduino) shortened the development, but the complication is still at the software level. Those boards are not intelligent enough to extract the knowledge from the raw data coming from sensors, and to make decision [79]. Figure 3 illustrates the current approach; the sensors collect the raw data from the environment around and send it to the gateway at the edge layer. Thereafter, the gateway converts the data between the protocols and forward the data to the cloud, Then, the cloud processes the data, extracts the knowledge and decides whether to actuate a thing or not. Finally, the cloud sends the decision to the actuator at the end devices layer.

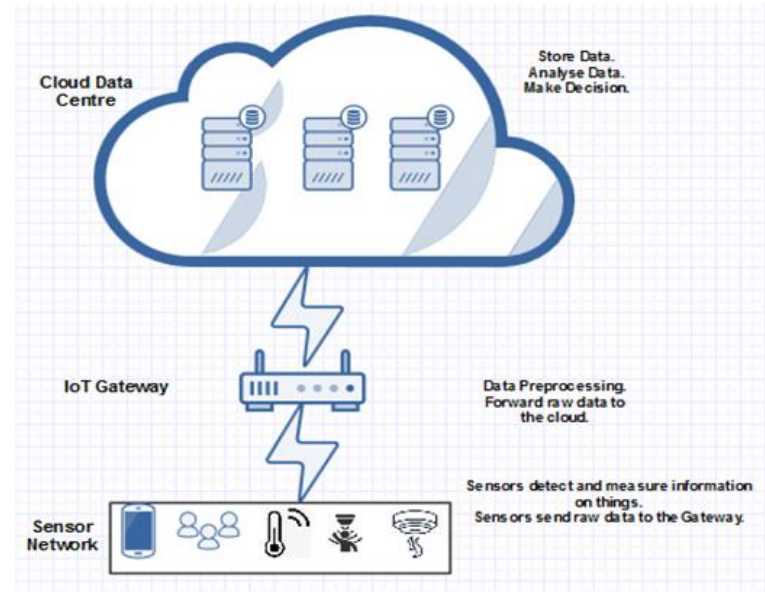

Figure 3: Existing model of the integration between cloud and IoT

This existing approach of sending all raw data generated by end devices to cloud using an IoT gateway is not efficient as it impacts the network's reliability, availability, robustness, and security and privacy. For example, with respect to availability, supposedly we have a smart, cloud-based home system, there is a fire inside the home, and there is no Internet connection. In this case, the system will fail to connect with the cloud to process the data and to take any action against the fire. Some contributions proposed a basic role for the IoT gateway [80], for example, applying predefined rules that are not efficient especially with complex IoT applications as they require many rules. IoT applications connect many things that make the intelligence by predefined rules fail to scale well. Furthermore, predefined rules are not able to offer intelligence in undefined conditions as they only offer presumed intelligence [81].

On the other hand, intelligence should not only reside in the cloud. Nowadays, there is a need for providing intelligence at both the cloud and edge layers. Depending on 
the situation, the decision should be made on the edge of the cloud to provide the desired functionality and performance, as shown in Figure 4. This can be applied by distributing the intelligence between the edge and the cloud layers [82]. Distributed intelligence in IoT means to distribute the intelligence over the edge and the cloud layers to provide the required functionality and performance for IoT applications. IoT gateway at the edge layer should be able to process the raw data and make decision, and then send only the necessary data to the cloud [83].

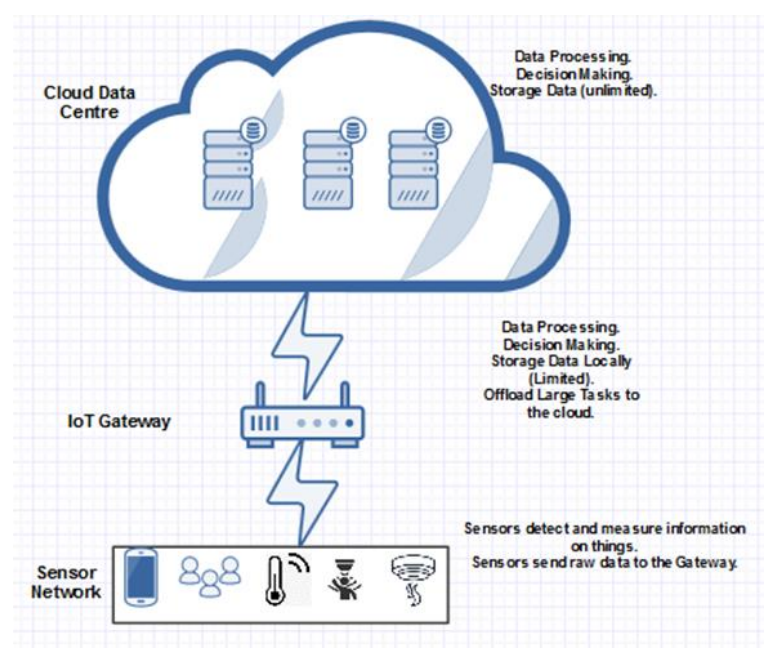

Figure 4: The distributed intelligence model

The question here is how to provide the gateway with the intelligence to extract the knowledge from the raw data? Algorithms and techniques should be running on IoT gateway, the algorithms should be able to process real-time raw data and extract the knowledge to make the decision. It should be able to perform its function even with the loss of cloud connectivity.

Machine learning can be a powerful solution for processing data produced by IoT devices. Combining machine learning and IoT gateway can help to analyze the data locally and to make the decision instead of sending the data to the cloud [84]. It also can provide real-time prediction, security, reliability, and availability. However, many IoT gateway controllers such as Raspberry Pi and Arduino have limited processing capabilities; hence, the gateway should use some techniques based on CPU utilization, CPU temperature, number of tasks, and type of application, to determine when to offload the task to the cloud. For example, the gateway might process only a certain number of coming tasks simultaneously and offload the others to the cloud.

The benefits of enabling Distributed Intelligence in IoT can be summarized as the followings:

- Local data processing of the most real-time data for fast decision and action made;

- Offload the complex tasks to the cloud;

- Reduce the data transmitted to the cloud for security and privacy purposes [85];

- Enable IoT networks to be more resourceful in terms of energy and communication bandwidth;

- To provide interoperability for heterogeneous platforms and communication protocols used in IoT; and

- Enable end devices to interact even with no connection with the cloud.

\section{IOT APPLiCATIONS REQUIRE DISTRIBUTED INTELLIGENCE}

\section{A. Smart Home}

In the past, the smart home was a science fiction idea. For example, Ray Bradbury imagined homes were interactive and ran themselves. It was terrifying until the actual benefits of home automation were considered by humans, and then the idea started to be more acceptable [61]. In the 19th century, many hardware and software emerged, leading to smart home applications. Also, the X10 standard was industrialized to allow sending and receiving broadcast messages via radiofrequency. Currently, the development of computer boards such as raspberry pi, Arduino, and FPGA helps scholars to build and develop inexpensive smart home applications. For example, Raspberry $\mathrm{Pi}$ is a cheap, smallsized computer that plugs into an LCD, keyboard, and mouse. It has different types of communication ports, such as Ethernet, USB ports, and HDMI port. It can perform all the functions of a desktop computer like word processing, browsing, and playing game.

Nowadays, most of the researches related to smart home applications aim to build a comfortable, secure, accessible, and safe system inside the home with the consideration of minimizing operating energy and cost. Sensors are attached to all targeted home appliances to be considered smart objects. They can sense, process data, and communicate. These devices from time to time carry out sensing and perform basic data processing, before sending the data to the hub, gateway or directly to the cloud. There are many challenges concerning smart home system design, but this paper only concern with Distributed Intelligence. In most of the smart home applications, all data need to be transferred to the cloud where the decision is made. This approach takes a long time and threatens security because the data are shared with the outside network. Furthermore, it will impact availability, if there is no Internet connection. On the other hand, some smart home applications can make the decision locally. However, those applications are predefined rules based, which is impractical with complex IoT applications. As mentioned in section IV, predefined rules fail to scale well and to offer intelligence in undefined conditions. Therefore, the home gateway should be provided with the intelligence that enable it to decide whether to make the decision locally or on the cloud. The gateway might use the following techniques to decide where to process the data: CPU utilization, CPU temperature, number of the running tasks, or the type of task. If it is locally, the gateway will be able to extract the knowledge from the raw data coming from the sensors and make the decisions without sending the data to the cloud-based on machine learning algorithms. Machine learning algorithms for processing data at the smart home gateway are required; it can make the decision and activation based on the analysis of experience.

In some cases, when the gateway is unable to make the decision, it will offload the task to the cloud. For example, when turning on the heating system at a smart home, the sensors will collect the data of temperature, time, and the motion at home, and then send it to the smart IoT gateway at the edge layer. The gateway will process the data, take the decision based on an intelligent algorithm to turn on/off the heating system, and send the decision back to the heating system at the home. However, if the task consumes the CPU, the gateway will forward the data to the cloud layer. For 
example, when a video processing task consumes the CPU, the gateway will offload it to the cloud layer.

\section{B. Internet of Vehicles (IoV)}

The IoV is a group of applications for Information processing and sharing to ease overcrowding, enhance traffic management, reduce the environmental influence, and increase revenue for transport to business users and the public [3]. It is an advanced technology with the integration of sensors, Internet, wireless communication, protocols for exchanging information among fast speed vehicles. The traditional IoV systems with central data processing at the cloud experience have many significant challenges such as connectivity and coverage, communication and energy cost, congestion, coordination and implementation, and traffic incident notification [66]. It cannot efficiently deal with realtime data [69], as each vehicle will transfer its generated data to the cloud through the Internet. A large number of vehicles will produce a huge amount of data that will impact the network. Furthermore, the traditional system may cause privacy problems because the current location of vehicles is shared. They also share the beginning and the end journey of tours with the cloud server [69]. Moreover, when there are several cars at a certain street contacting the cloud to avoid the traffic, the cloud will ask all those cars to move to a street that is parallel to the first one. This will shift the traffic from one street to another.

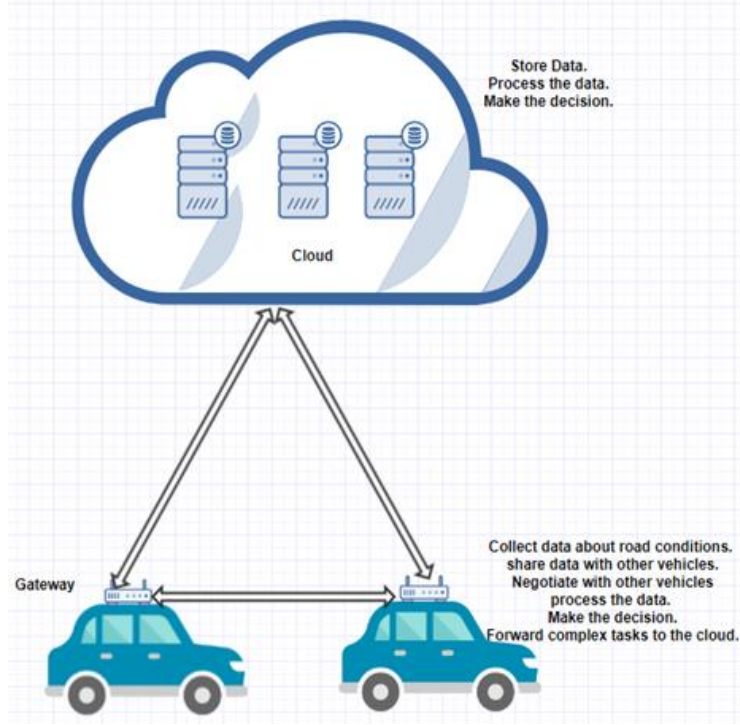

Figure 5: Smart IoV

The IoV should be able to process real-time data efficiently without sending all of them to the cloud, as shown in Figure 5 . This can be achieved by bringing part of the intelligence to the edge layer and make it able to decide by extracting the knowledge from raw data collected from sensors. Each vehicle has to be attached to a smart gateway that connects it with other vehicles in the same area to share the data about road and traffic conditions. The gateway should be provided with the intelligence to process the data and to make the decision. Applying this scenario will improve the availability of the system, as it will be able to make the decision even with no connection with the cloud.

Enabling Distributed Intelligence between the cloud and a smart gateway in IoV will reduce the delay and enhance the safety of IoV applications. One case is re-routing vehicles in a traffic management system to avoid congestion needs the information to be shared between vehicles from different road areas [86]. The smart gateway attached to the vehicle should collect the data of the traffic condition and negotiate with the other gateways of vehicles on the road to decide to either reroute the vehicle or not.

\section{Key CHALLENGES}

Although Distributed Intelligence will improve the performance of IoT applications, there are many research challenges and questions. This section illustrates the challenges of enabling Distributed Intelligence between cloud computing and edge computing with some suggested solutions, such as: How the gateway and the cloud interact with each other? Which tasks should be offloaded to the cloud? Which techniques determine to either process the task locally or offload it to the cloud?

\section{A. Single Board Computer Capabilities}

Nowadays, single board computers have been developed rapidly. However, they still have limited computations and storage capabilities that impact their role to be a gateway between the end devices and the cloud layers, as end devices generate a huge amount of data that requires processing. The gateway has to be robust and flexible to take care of several issues such as data processing, data management, security, and QoS. It is challenging to accomplish all the tasks instantaneously using current resource-constrained single board computers. Thus, the solution is to offload certain tasks to the cloud for processing and making decision. Offloading techniques will keep the gateway under load and perform their jobs properly.

\section{B. IoT Gateway Platform Issues}

The software frameworks at the gateway level still have challenges in processing raw data locally and taking action. An intelligence algorithm should be implemented in the platform to be able to process data based on learning from experience. Combining IoT and machine learning is essential to provide intelligence at the gateway to learn from experience. Many machine learning algorithms can play an essential role in processing IoT data such as decision trees, deep learning, and reinforcement learning.

\section{Tasks Management}

Distributed Intelligence aims to distribute the tasks between the gateway and the cloud. The question here is which factors govern the distribution of tasks? The computation tasks can be distributed in two scenarios. The first one is to process simple tasks locally in the gateway and offload complex tasks to the cloud. The second one is to use system metrics of the gateway such as CPU and RAM utilization to decide either to offload the task or process the tasks locally. For example, when the CPU utilization of the gateway is over $75 \%$, offload all-new coming tasks to the cloud can be considered.

\section{CONCLUSION}

This work has presented a deep investigation of Distributed Intelligence under IoT. It is a paradigm to distribute the intelligence over the edge and cloud layers to provide the required functionality and performance for IoT applications. 
Distributed Intelligence in IoT plays an important role to support the exact communication and processing functions to be offered at the exact place at a certain time. This can be achieved by integrating cloud and edge computing in one model and providing the edge with a smart gateway that has two main jobs: Firstly, deciding to process the data locally or to send it to the cloud, and secondly, processing the local data to extract the knowledge and to make decision. Distributed Intelligence in IoT is related to Artificial intelligence, IoT, and Cloud Computing. Therefore, corporations among scholars of those areas should propose and standardize certain Distributed Intelligence paradigms for the popular IoT applications such as smart homes, intelligence of vehicles, and intelligence watering system.

\section{REFERENCES}

[1] Networking, Cisco Visual. "Cisco Global Cloud Index: Forecast and Methodology, 2015-2020. White paper." Cisco Public, San Jose (2016).

[2] Sahni, Yuvraj, et al. "Edge Mesh: A new paradigm to enable distributed intelligence in Internet of Things." IEEE Access 5 (2017): 16441-16458.

[3] Alam, Tanweer. "Fuzzy control based mobility framework for evaluating mobility models in MANET of smart devices", ARPN Journal of Engineering and Applied Sciences, 12(15), (2017) pp.: 4526-4538

[4] Al-Fuqaha, Ala, et al. "Internet of things: A survey on enabling technologies, protocols, and applications." IEEE Communications Surveys \& Tutorials 17.4 (2015): 2347-2376.

[5] Tanweer Alam, "Efficient and Secure Data Transmission Approach in Cloud-MANET-IoT integrated Framework", Journal of Telecommunication, Electronic and Computer Engineering (JTEC) Vol. 12 No. 1, 2020.

[6] Tanweer Alam, "Internet of Things: A Secure Cloud-Based MANET Mobility Model", International Journal of Network Security, Vol. 22(3), 2020.

[7] Tanweer Alam, Yazeed Mohammed Alharbi, Firas Adel Abusallama, Ahmad Osama Hakeem, "Smart Campus Mobile Application Toward the Development of Smart Cities", International Journal of Applied Sciences and Smart Technologies, Vol. 2 (1), 2020.

[8] Tanweer Alam, "A Middleware Framework between Mobility and IoT Using IEEE 802.15.4e Sensor Networks", Jurnal Online Informatika, Vol 4, No 2 (2019). DOI: https://doi.org/10.15575/join.v4i2.487

[9] Parker, Lynne E. " Distributed intelligence: Overview of the field and its application in multi-robot systems." Journal of Physical Agents 2.1 (2008): 5-14.

[10] K. Kevin., "That 'internet of things' thing," RFID journal, vol. 22, no. 7, pp. 97-114, 2009.

[11] Rao, B. Prahlada, P. Saluia, N. Sharma, A. Mittal and S. V. Sharma "Cloud computing for Internet of Things \& sensing based applications," in In Sensing Technology (ICST), 2012 Sixth International Conference on, 2012.

[12] S. Babu, C. M, L. P, K. Ganapathy and V. Vaidehi, "Cloud-Enabled Remote Health Monitoring System," in 2013 International Conference on Recent Trends in Information Technology (ICRTIT), 2013.

[13] R. Yu, Y. Zhang and S. Gjessing, "Toward Cloud-based Vehicular Networks with Efficient Resource Management," IEEE Network, vol. 27, no. 5, pp. 48-55, 2013.

[14] M. Hassanalieragh, A. Page, T. Soyata, G. Sharma, M. Aktas, G. Mateos, B. Kantarci and S. Andreescu, "Health Monitoring and Management UsingInternet-of-Things (IoT) Sensing with CloudbasedProcessing: Opportunities and Challenges," in 2015 IEEE International Conference on Services Computing, 2015.

[15] Bagula, C. Lubamba, M. Mandava, H. Bagula, M. Zennaro and E. Pietrosemoli, "Cloud based patient prioritization as service in public health care," in 2016 ITU Kaleidoscope Academic Conference, 2016.

[16] Darwish, A. E. Hassanien, M. Elhoseny, A. K. Sangaiah and K. Muhammad, "The impact of the hybrid platform of internet of things and cloud computing on healthcare systems: opportunities, challenges, and open problems," Journal of Ambient Intelligence and Humanized Computing, pp. 1-16, 2017.

[17] W. Paper, "ArFog Computing and the Internet of Things: Extend the the Cloud to Where the Things," 2015.
[18] Bonomi, Flavio, R. Milito, J. Zhu and S. Addepalli, "Fog computing and its role in the internet of things," in Proceedings of the first edition of the MCC workshop on Mobile cloud computing, 2012.

[19] Y.-Y. Shih, W.-H. Chung, A.-C. Pang, T.-C. Chiu and H.-Y. We, "Enabling low-latency applications in fog-radio access networks," IEEE network, vol. 31, no. 1, pp. 52-58, 2017

[20] Z. Ning, X. Kong, F. Xia, W. Hou and X. Wang, "Green and sustainable cloud of things: Enabling collaborative edge computing," IEEE Communications Magazine, vol. 75, no. 1, pp. 72-78, 2018.

[21] Cavalcante, Everton, J. Pereira, M. P. Alves, P. Maia, R. Moura, T. Batista, F. C. Delicato and P. F. Pires, "On the interplay of Internet of Things and Cloud Computing," Computer Communications, vol. 89, pp. 17-33, 2016.

[22] M. Aazam, P. P. Hung and E.-N. Huh, "Cloud of Things: Integrating Internet of Things withCloud Computing and the Issues Involved," Proceedings of 2014 11th International Bhurban Conference on Applied Sciences \& Technology (IBCAST), pp. 414-419, 14th-18th January January 2014.

[23] Babu, S. Masthan, A. J. Lakshmi and a. B. T. Rao, "A study on cloud based Internet of Things: CloudIoT," in 2015 Global Conference, 2015.

[24] F. Li, M. V. ogler, M. Claeßens and S. Dustdar, "Towards Automated IoT Application Deploymentby a Cloud-based Approach," 2013 IEEE 6th International Conference on Service-Oriented Computing and Applications, 2013.

[25] Khodkari, Hourieh, S. Maghrebi, and Rey Branch. "Necessity of the integration Internet of Things and cloud services with quality of service assurance approach." Bulletin de la Société Royale des Sciences de Liège 85, no. 1 (2016): 434-445.

[26] R. Mueller, J. S. Rellermeyer, M. Duller and G. Alonso, “A generic platform for sensor network applications.," in 2007 IEEE International Conference on Mobile Adhoc and Sensor Systems., 2007.

[27] J.-W. Yoon, Y.-k. Ku, C.-S. Nam and D.-R. Shin, "Sensor network middleware for distributed and heterogeneous environments.," in 2009 International Conference on New Trends in Information and Service Science., 2009.

[28] Bimschas, H. Hellbrück, R. Mietz, D. Pfisterer, K. Römer and T. Teubler, "Middleware for smart gateways connecting sensornets to the internet.," in Proceedings of the 5th International Workshop on Middleware Tools, Services and Run-Time Support for Sensor Networks., 2010.

[29] S. Guoqiang, C. Yanming, Z. Chao and Z. Yanxu., "Design and Implementation of a Smart IoT Gateway," in 2013 IEEE International Conference on Green Computing and Communications and IEEE Internet of Things and IEEE Cyber, 2013.

[30] J. Bian, D. Fan and J. Zhang., "The new intelligent home control system based on the dynamic and intelligent gateway," in In 2011 4th IEEE International Conference on Broadband Network and Multimedia Technology, 2011.

[31] W. Shen, Y. Xu, D. Xie, T. Zhang and A. Johansson., "Smart border routers for ehealthcare wireless sensor networks," in In 2011 7th International Conference on Wireless Communications, Networking and Mobile Computing, 2011.

[32] V. Stantchev, A. Barnawi, S. Ghulam, J. Schubert and G. Tamm., "Smart Items, Fog and Cloud Computing as Enablers of Servitization in Healthcare," Sensors \& Transducers, vol. 185, no. 2, p. 121, 2015.

[33] M. Rahmani, T. N. Gia, B. Negash, A. Anzanpour, I. Azimi, M. Jiang and P. Liljeberg, "Exploiting smart e-Health gateways at the edge of healthcare nternet-of-Things: A fog computing approach," Future Generation Computer Systems, 2017.

[34] Azimi, A. Anzanpour, A. M. Rahmani, T. Pahikkala, M. Levorato, P. Liljeberg and N. Dutt, "HiCH: Hierarchical Fog-assisted Computing Architecture for Healthcare IoT," ACM Transactions on Embedded Computing Systems (TECS), vol. 16, no. 5s, p. 174, 2017.

[35] Corporation, "An architectural blueprint for autonomic computing," White paper, 2006.

[36] P. Wang, F. Ye and X. Chen, "A Smart Home Gateway Platform for Data Collection and Awareness," IEEE Communications Magazine, vol. 56, no. 9, pp. 87-93, 2018.

[37] H. Rahman and R. Rahmani, "Enabling distributed intelligence assisted Future Internet of Things Controller (FITC)," Applied Computing and Informatics, vol. 4, no. 1, pp. 73-87, 2018.

[38] M. Rahmani, T. N. gia, B. S. Negash, A. Anzanpour, I. Azimi, M Jiang and P. Liljeberg, "Exploiting Smart E-Health Gateways at the Edge of Healthcare Internet-of-Things: A Fog Computing Approach," Future Generation Computer Systems, 2017.

[39] Saleh, Nayif, Abdallah Kassem, and Ali M. Haidar. "Energy-Efficient Architecture for Wireless Sensor Networks in Healthcare Applications." IEEE Access 6 (2018): 6478-6486. 
[40] Rawat, Priyanka, Kamal Deep Singh, Hakima Chaouchi, and Jean Marie Bonnin. "Wireless sensor networks: a survey on recent developments and potential synergies." The Journal of supercomputing 68, no. 1 (2014): 1-48.

[41] Eisenhauer, Markus, Peter Rosengren, and Pablo Antolin. "A development platform for integrating wireless devices and sensors into ambient intelligence systems." In Sensor, Mesh and Ad Hoc Communications and Networks Workshops, 2009. SECON Workshops' 09. 6th Annual IEEE Communications Society Conference on, pp. 1-3. IEEE, 2009.

[42] Fernando, Niroshinie, Seng W. Loke, and Wenny Rahayu. "Mobile cloud computing: A survey." Future generation computer systems 29, no. 1 (2013): 84-106.

[43] Alam, T. Cloud Computing and Its Role in the Information Technology. IAIC Transactions on Sustainable Digital Innovation (ITSDI) 2020, $\quad 1, \quad 108-115 . \quad$ DOI https://doi.org/10.34306/itsdi.v1i2.103

[44] T. Alam "Design a blockchain-based middleware layer in the Internet of Things Architecture," JOIV: International Journal on Informatics Visualization, vol. 4, no. 1, Feb. 2020. https://doi.org/10.30630/joiv.4.1.334

[45] Xu, Ke, Yi Qu, and Kun Yang. "A tutorial on the internet of things: From a heterogeneous network integration perspective." IEEE Network 30, no. 2 (2016): 102-108.

[46] Dohr, Angelika, Robert Modre-Opsrian, Mario Drobics, Dieter Hayn, and Günter Schreier. "The internet of things for ambient assisted living." In Information Technology: New Generations (ITNG), 2010 Seventh International Conference on, pp. 804-809. Ieee, 2010.

[47] Spiess, Patrik, Stamatis Karnouskos, Dominique Guinard, Domnic Savio, Oliver Baecker, Luciana Moreira Sá De Souza, and Vlad Trifa "SOA-based integration of the internet of things in enterprise services." In Web Services, 2009. ICWS 2009. IEEE International Conference on, pp. 968-975. IEEE, 2009.

[48] Jia, Xiaolin, Quanyuan Feng, Taihua Fan, and Quanshui Lei. "RFID technology and its applications in Internet of Things (IoT)." In Consumer Electronics, Communications and Networks (CECNet), 2012 2nd International Conference on, pp. 1282-1285. IEEE, 2012.

[49] Lee, Youngjae, Jinhong Kim, W. Yu, and Kiyoung Moon. "An RFIDbased real-time localization of multiple AGV (Automatic Guided Vehicle) system for the guided path navigation." In Inter. Conf. on Indoor Positioning and Indoor Navigation (IPIN), Spain, pp. 1-2 2016.

[50] Windmann, Stefan, Oliver Niggemann, Holger Ruwe, and Friedrich Becker. "A novel self-configuration method for RFID systems in industrial production environments." In Emerging Technologies and Factory Automation (ETFA), 2017 22nd IEEE International Conference on, pp. 1-5. IEEE, 2017.

[51] Wang, Shiyong, Jiafu Wan, Di $\mathrm{Li}$, and Chunhua Zhang. "Implementing smart factory of industrie 4.0: an outlook." International Journal of Distributed Sensor Networks 12, no. 1 (2016): 3159805 .

[52] Rodríguez, Schubert, Tatiana Gualotuña, and Carlos Grilo. "A System for the Monitoring and Predicting of Data in Precision Agriculture in a Rose Greenhouse Based on Wireless Sensor Networks." Procedia Computer Science 121 (2017): 306-313.

[53] Saha, Rajesh, Santanu Maity, and Nripendra Trigunayat. "Enhancementof gain, bandwidth and directivity of a patch antenna by increasing dielectric layers of the substrate through micromachining technique for RFID application." In Computer Engineering and Applications (ICACEA), 2015 International Conference on Advances in, pp. 321-324. IEEE, 2015.

[54] Prabhu, Boselin, N. Balakumar, and A. Johnson Antony. "Evolving constraints in military applications using wireless sensor networks." (2017).

[55] Li, Heng, Greg Chan, Johnny Kwok Wai Wong, and Martin Skitmore. "Real-time locating systems applications in construction." Automation in Construction 63 (2016): 37-47.

[56] Gilligan, R., Susan Thomson, Jim Bound, and W. Stevens. Basic socket interface extensions for IPv6. No. RFC 2553. 1999.

[57] Montenegro, Gabriel, Nandakishore Kushalnagar, Jonathan Hui, and David Culler. Transmission of IPv6 packets over IEEE 802.15. 4 networks. No. RFC 4944. 2007.

[58] Thubert, Pascal, and Jonathan W. Hui. "Compression format for IPv6 datagrams over IEEE 802.15. 4-based networks." (2011).

[59] Bandyopadhyay, Soma, Munmun Sengupta, Souvik Maiti, and Subhajit Dutta. "A survey of middleware for internet of things." In Recent trends in wireless and mobile networks, pp. 288-296. Springer, Berlin, Heidelberg, 2011.
[60] Park, Namje, Hongxin Hu, and Qun Jin. "Security and privacy mechanisms for sensor middleware and application in internet of things (IoT)." (2016): 2965438.

[61] Alam, Tanweer. "Middleware Implementation in Cloud-MANET Mobility Model for Internet of Smart Devices." IJCSNS 17(5) (2017): 86-94.

[62] Alam, Tanweer, and B. K. Sharma. "A New Optimistic Mobility Model for Mobile Ad Hoc Networks." International Journal of Computer Applications 8, no. 3 (2010): 1-4. Doi: https://doi.org/10.5120/1196-1687

[63] Alam T, Benaida M. "The Role of Cloud-MANET Framework in the Internet of Things (IoT)", International Journal of Online Engineering (iJOE). Vol. 14(12), pp. 97-111. DOI https://doi.org/10.3991/ijoe.v14i12.8338

[64] Reiners, René, Andreas Zimmermann, Marc Jentsch, and Yan Zhang. "Automizing home environments and supervising patients at home with the hydra middleware: application scenarios using the hydra middleware for embedded systems." In Proceedings of the first international workshop on Context-aware software technology and applications, pp. 9-12. ACM, 2009.

[65] Yang, Yiling, Yu Huang, Xiaoxing Ma, and Jian Lu. "Enabling context-awareness by predicate detection in asynchronous environments." IEEE Transactions on Computers 65, no. 2 (2016) 522-534.

[66] Alam T, Benaida M. CICS: Cloud-Internet Communication Security Framework for the Internet of Smart Devices. International Journal of Interactive Mobile Technologies (iJIM). 2018 Nov 1;12(6):74-84. DOI: https://doi.org/10.3991/ijim.v12i6.6776

[67] Armbrust, Michael, Armando Fox, Rean Griffith, Anthony D. Joseph, Randy Katz, Andy Konwinski, Gunho Lee et al. "A view of cloud computing." Communications of the ACM 53, no. 4 (2010): 50-58.

[68] Zhang, Qi, Lu Cheng, and Raouf Boutaba. "Cloud computing: stateof-the-art and research challenges." Journal of internet services and applications 1, no. 1 (2010): 7-18.

[69] Tanweer Alam, "Blockchain and its Role in the Internet of Things (IoT)", International Journal of Scientific Research in Computer Science, Engineering and Information Technology, vol. 5(1), pp. 151157, 2019. DOI: https://doi.org/10.32628/CSEIT195137

[70] Tanweer Alam, Baha Rababah, "Convergence of MANET in Communication among Smart Devices in IoT", International Journal of Wireless and Microwave Technologies (IJWMT), Vol.9, No.2, pp. 1-10, 2019. DOI: 10.5815/ijwmt.2019.02.01

[71] Botta, Alessio, Walter De Donato, Valerio Persico, and Antonio Pescapé. "Integration of cloud computing and internet of things: a survey." Future Generation Computer Systems 56 (2016): 684-700.

[72] Tanweer Alam, "IoT-Fog: A Communication Framework using Blockchain in the Internet of Things", International Journal of Recent Technology and Engineering (IJRTE), Volume-7, Issue-6, 2019.

[73] Whaiduzzaman, Md, Mehdi Sookhak, Abdullah Gani, and Rajkumar Buyya. "A survey on vehicular cloud computing." Journal of Network and Computer Applications 40 (2014): 325-344.

[74] Al Nuaimi, Klaithem, Nader Mohamed, Mariam Al Nuaimi, and Jameela Al-Jaroodi. "A survey of load balancing in cloud computing: Challenges and algorithms." In Network Cloud Computing and Applications (NCCA), 2012 Second Symposium on, pp. 137-142. IEEE, 2012.

[75] Rahimi, M. Reza, Jian Ren, Chi Harold Liu, Athanasios V. Vasilakos, and Nalini Venkatasubramanian. "Mobile cloud computing: A survey, state of art and future directions." Mobile Networks and Applications 19, no. 2 (2014): 133-143.

[76] Ryan, Mark D. "Cloud computing security: The scientific challenge, and a survey of solutions." Journal of Systems and Software 86, no. 9 (2013): 2263-2268

[77] Beck, Michael Till, and Marco Maier. "Mobile edge computing Challenges for future virtual network embedding algorithms." In Proc. The Eighth International Conference on Advanced Engineering Computing and Applications in Sciences (ADVCOMP 2014), vol. 1, no. 2, p. 3. 2014.

[78] Mao, Yuyi, Changsheng You, Jun Zhang, Kaibin Huang, and Khaled B. Letaief. "A survey on mobile edge computing: The communication perspective." IEEE Communications Surveys \& Tutorials 19, no. 4 (2017): 2322-2358

[79] Handoyo, Ikut Tri, and Dana Indra Sensuse. "Knowledge-based systems in decision support context: A literature review." New Media Studies (CONMEDIA), 2017 4th International Conference on. IEEE, 2017.

[80] Mansour Bader, Andraws Swidan, Mazin Al-Hadidi, and Baha Rababah. "A Binary to Residue Conversion Using New Proposed Non-Coprime Moduli Set." Signal \& Image Processing: An International Journal (SIPIJ) Vol 7(3), 2016. 
[81] Borgia, Eleonora. "The Internet of Things vision: Key features, applications and open issues." Computer Communications 54 (2014): $1-31$.

[82] Bill McBeath, "Distributed Intelligence in the IoT", chainlink, URL: http://www.clresearch.com/research/detail.cfm?guid=4E088DB8 3048-79ED-9964-69B73742171B

[83] Sanaei, Zohreh, Saeid Abolfazli, Abdullah Gani, and Rajkumar Buyya. "Heterogeneity in mobile cloud computing: taxonomy and open challenges." IEEE Communications Surveys \& Tutorials 16, no. 1 (2014): 369-392.
[84] Liu, Wei, Qiang Liu, and Wensu Li. "Research of IoT Application in Manned Spaceflight Launch Site." In Wireless Communications, Networking and Applications, pp. 517-527. Springer, New Delhi, 2016.

[85] Roman, Rodrigo, Jianying Zhou, and Javier Lopez. "On the features and challenges of security and privacy in distributed internet of things." Computer Networks 57, no. 10 (2013): 2266-2279.

[86] Das, Aditi, and Ravi Prakash Gorthi. "Knowledge Based Routing Protocols in Wireless Sensor Networks." In Computational Intelligence, Communication Systems and Networks (CICSyN), 2015 7th International Conference on, pp. 35-38. IEEE, 2015. 